\title{
Fachbeitrag
}

Babett Goldstein und Antje Meye

\section{Bearbeitung von monografischen E-Ressourcen über besondere Erwerbungsmodelle in Alma}

http://doi.org/10.1515/abitech-2018-2003

Zusammenfassung: Alma bietet für Erwerbungsmodelle von E-Ressourcen eine Vielzahl an Bearbeitungsmöglichkeiten. Zum einen können Metadaten von Verlagen komfortabel aufbereitet und eingespielt werden - einschließlich der Erzeugung eines elektronischen Bestandes (Portfolio) - und zum anderen können Metadatensätze auf Verbundebene (B3Kat) genutzt und mit Linking-Informationen aus der Community Zone verknüpft werden. Diese Vielseitigkeit erspart nicht nur Doppelarbeit in den unterschiedlichen Systemen wie SFX oder Verde, sondern ermöglicht jeder Bibliothek auch, auf die neuen Anforderungen für E-Ressourcen schnell und zeitgemäß zu reagieren. Der Aufsatz beschreibt Erwerbungsmodelle für monografische E-Ressourcen und deren Handhabung an zwei Berliner Universitätsbibliotheken, die Alma benutzen.

Schlüsselwörter: Erwerbungsmodelle, PDA-Modell, EBSModell

\section{Alma offers a variety of options for using new acquisition models of electronic resources}

On the one hand, the metadata provided by publishers can be managed and loaded comfortably - including the option to create an electronic portfolio; on the other hand, metadata from the B3Kat (where we do cooperative cataloguing within the Bavarian Library Network) can be used and connected with linking-information from the Community Zone. The great versatility does not only avoid the duplication of work in different systems such as SFX or Verde, it also allows every library to react quickly and appropriately to new demands for electronic resources. The article describes the acquisition methods for monographic electronic resources and their handling in two Berlin university libraries using Alma.

Keywords: Acquisition models, patron-driven acquisition, evidence-based selection

\section{Benutzergesteuerte Erwerbung in Alma (PDA) an der HU Berlin}

Ein wichtiges Feld für den effizienten Einsatz des Bibliothekssystems Alma ist die Erwerbung von E-Books in der sogenannten nutzergesteuerten Erwerbung, z. B. die Patron-Driven-Acquisition (PDA) bzw. Demand-DrivenAcquisition (DDA). Dabei wird über einen Aggregator eine Vielzahl an Titeln verschiedener Verlage bereitgestellt.

Diese Titel wurden vor der Einführung von Alma aus einer Gesamtdatei direkt in das Suchportal Primo eingespielt. Die notwendige Dublettenprüfung wurde anhand der gelieferten Daten vom Aggregator auf dem Linkresolver SFX anhand von Excel-Tabellen durchgeführt. Nur auf der Aggregatoren-Plattform war zu erkennen, in welcher Fachkollektion ein Titel freigeschaltet wurde. Erst nachdem durch mehrmalige Nutzung ein Kauf ausgelöst wurde, sind die Titel im Verbund B3Kat katalogisiert und mit einem Besitzkennzeichen versehen worden. Im Anschluss wurde die URL für den Zugriff im Lokalsystem eingetragen und eine nachträgliche Bestellung angelegt. Anfangs war nur durch eine zusätzlich erfasste Information ersichtlich, mit welchem Guthaben aus welcher Kollektion das E-Book gekauft wurde. Pausierte eine Kollektion oder wurde sie beendet, mussten die jeweiligen Titel aus Primo entfernt werden. Aufgrund der Arbeitsverteilung auf Kolleginnen und Kollegen in unterschiedlichen Abteilungen dauerte dies allerdings ein paar Tage.

Mit der Einführung von Alma sind die Arbeitsabläufe für die Erwerbungsart PDA nun an einer Stelle möglich. Die Überschneidungsanalyse in Alma bietet vorab die Möglichkeit zu prüfen, welche Titel bereits im System vorhanden sind. Für die einzelnen Kollektionen werden mit dem Tool „Von Nutzern veranlasste Erwerbung (PDA)“ jeweils eigene Projekte angelegt (Abb. 1).

Diese können mit einem Start- und Enddatum versehen sowie mit einem Guthaben-Schwellenwert vorbelegt werden. In einem weiteren Arbeitsschritt werden die vorliegenden MARC-Daten über den PDA-Bestand eingespielt. Nach Bedarf können die einzelnen Projekte pausieren oder komplett bereinigt werden. Ist ein Enddatum vorgegeben, werden die Portfolios zu diesem Zeitpunkt auto- 
Von Benutzern veranlasste Erwerbungen (PDA)

\begin{tabular}{|c|c|c|c|c|c|c|c|c|c|}
\hline \multicolumn{2}{|c|}{$1-20$ of 24} & Alle - & & Q & & & († Neue PDA & $\leftrightarrow$ & 0 \\
\hline & \multicolumn{9}{|c|}{$\boldsymbol{\nabla}$ Filter: Alle } \\
\hline & $\triangle \mathrm{Nam}$ & & $\rightarrow$ Code & Status & Lieferant & 今 Startdatum & $\rightarrow$ Enddatum & & \\
\hline 1 & $\begin{array}{l}\text { Cambric } \\
\text { EBS-Pro }\end{array}$ & $\begin{array}{l}\text { dge University Press } \\
\text { jekt }\end{array}$ & cup_all & Aktiv & Cambridge University Press & $01 / 01 / 2018$ & $31 / 12 / 2018$ & & $\cdots$ \\
\hline 2 & $\begin{array}{l}\text { Duncker } \\
\text { Projekt }\end{array}$ & $\begin{array}{l}\text { \& \& Humblot NBE- } \\
\text { Recht }\end{array}$ & d\&h_rew & Aktiv & $\begin{array}{l}\text { Duncker \& Humblot GmbH, } \\
\text { Verlagsbuchhandlung }\end{array}$ & $01 / 01 / 2018$ & $31 / 01 / 2019$ & & $\cdots$ \\
\hline 3 & Ebrary A & Agrarwissenschaft & ebr_agr & Unterbrochen & $\begin{array}{l}\text { Lehmanns Media GmbH/E- } \\
\text { Book-Team }\end{array}$ & 19/01/2017 & - & & $\cdots$ \\
\hline
\end{tabular}

Abb. 1: Kollektion „Von Benutzern veranlasste Erwerbungen (PDA)“

matisch deaktiviert. Nach dem Kauf eines E-Books wird an dem jeweiligen Portfolio eine Bestellung angelegt. In den Erwerbungsinformationen ist sofort ersichtlich, über welche Kollektion der Titel erworben wurde. Ist ein Projekt abgeschlossen und sind für die gekauften E-Books Bestellungen angelegt, kann das Projekt geschlossen werden. Die nicht gekauften Portfolios werden automatisch aus Alma entfernt.

\section{Evidence Based Selection (EBS) und E-Book-Paketkauf an der FU Berlin}

Bei diesem Modell wird der Bibliothek der Zugriff auf die komplette Verlagsplattform für einen definierten Zeitraum gewährt, anschließend werden anhand von Nutzungsstatistiken E-Book-Käufe in Höhe eines zuvor hinterlegten Depots ausgelöst.

Auch hier wird hauptsächlich die Möglichkeit von Importprofilen für die Verwaltung von EBS-Modellen genutzt. Damit konnten mit dem Umstieg auf Alma seit 2017 acht verschiedene EBS-Modelle mit insgesamt 260000 E-Book-Titeldaten verwaltet werden. Entweder gab es ein geeignetes Paket (elektronische Sammlung) mit den entsprechenden Portfolios in der Community Zone, die aktiviert werden konnte, oder man hat die vom Verlag gelieferten MARC-Titeldaten mit einem eigens für das EBS-Modell konfigurierten Importprofil nach Alma eingespielt (Abb. 2). Im vordefinierten Zeitraum können mit diesen Importprofilen auch zeitnah Updates von neu erschienenen E-Books ins Alma-System eingespielt werden. Alle EBS-Kollektionen werden dann mit einem Deaktivierungsdatum versehen, so dass automatisch nach Ablauf des Zugriffs (in der Regel ein Jahr) alle Portfolios in Alma deaktiviert und ggf. die Metadaten gelöscht werden, damit sie nicht mehr im Discovery-System Primo recherchierbar sind.

Die daraus resultierenden E-Book-Käufe (anhand von Nutzungsstatistiken) werden dann einzeln über den B3KAT katalogisiert und in Alma als E-Book-Kauf nachgewiesen.

Bei den gekauften E-Book-Paketen (wie z.B. vom Springer-Verlag) wird auf die Bereitstellung der Metadaten auf Verbundebene zurückgegriffen, das heißt, die Verbundzentrale setzt im B3KAT die Besitzkennzeichen an die von der FU lizenzierten Springer-Pakete, und über die VSTSchnittstelle kommen die Titelmetadaten automatisch in das Alma-System. Lokale Kennzeichen, wie z. B. das ZDBProduktsigel, dienen dann als Kriterium, um diese Meta-

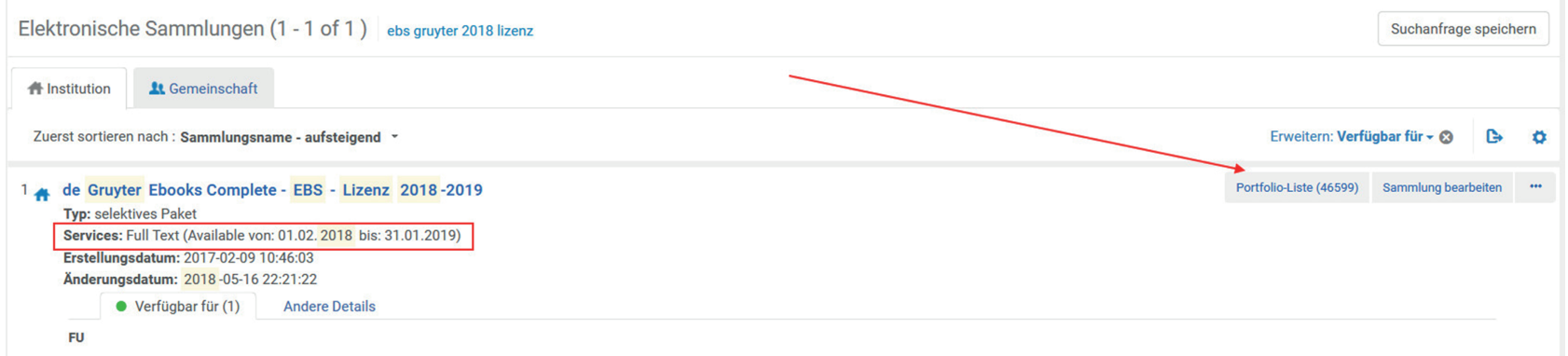

Abb. 2: Lokale Elektronische Sammlung mit Enddatum und Anzahl der dazugehörigen Portfolios 
$<$ Importprofil-Details

springer deutsch zdb-2-SWI- 2018

Profiltyp Bestand

\begin{tabular}{l|l|l|l} 
Profildetails Normierung \& Überprüfung & Match-Profil & Satzkennzeichen einstellen & Bestandsinformationen
\end{tabular}

Bestandsvorgänge

Electronic

E-Book Zuordnung

Portfolios löschen/deaktivieren Nein

Portfoliotyp Teil einer elektronischen Sammlung

Elektronische Sammlung Springer Ebook Collection - Wirtschaftswissenschaften deutsch - 2018

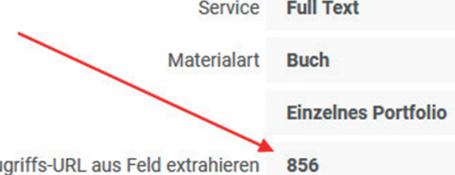

Zugriffs-URL aus Feld extrahieren 856

Abb. 3: Ausschnitt aus dem Importprofil für Springer-E-Books

daten entsprechend den jeweiligen E-Book-Paketen in verschiedene Sets zu gruppieren. Das nur aus Metadaten bestehende Set wird dann als MARC-Datei exportiert und erneut über ein Importprofil mit einem definierten MergeProzess eingespielt, so dass Portfolios gebildet und diese wiederum der korrekten elektronischen Sammlung „zugeordnet" werden (Abb. 3).

Abschließend werden die lokal in Alma angelegten Sammlungen mit der Community Zone verknüpft. Damit gewährleistet die FU bei ihren Springer-Paketen, dass die Metadaten über die Verbundebene aktualisiert (Normdatenverknüpfung, Sacherschließung usw.) und zugleich aktuelle Linking-Informationen aus der Community Zone genutzt werden können.

Für die heute stark verbreiteten Modelle der Erwerbung von monografischen E-Ressourcen bietet Alma somit adäquate Funktionalitäten, die den Erwerbungsvorgang erheblich effektiver gestalten.

\section{Autoreninformationen}

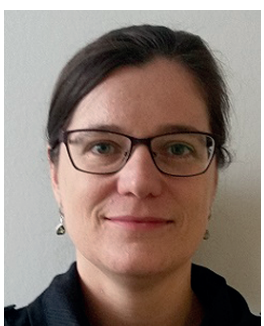

Babett Goldstein

Abteilung für Medienerwerbung und -erschließung

Humboldt-Universität zu Berlin

Universitätsbibliothek

Unter den Linden 6

10099 Berlin

babett.goldstein@ub.hu-berlin.de

orcid.org/0000-0001-7030-2280

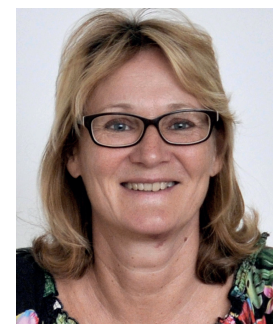

Antje Meye

Abteilung Zugang - Monographien

Freie Universität Berlin

Universitätsbibliothek

Garystr. 39

14195 Berlin

meye@ub.fu-berlin.de

orcid.org/0000-0002-3976-4839 